\title{
In situ Comparative heating and simultaneous multi-detector imaging at high and ultra-low landing energies
}

Atsushi Muto ${ }^{1}$, Stas Dogel ${ }^{2}$, Kotaro Hosoya ${ }^{2}$, Hooman Hosseinkhannazer ${ }^{3}$, Najia Mahdi ${ }^{3}$ and Michael T Postek $^{4}$

${ }^{1}$ Hitachi High-Tech America, Clarksburg, Maryland, United States, ${ }^{2}$ Hitachi High-Tech Canada, Inc., United States, ${ }^{3}$ Norcada, Inc., United States, ${ }^{4}$ University of South Florida, United States

Rapid improvements in many phases of scanning electron microscope electronics enables simultaneous imaging and correlation using multiple electron detectors of materials under test, especially those using in situ heating stages. Often, the results of these experiments appear rapidity, and thus collecting and recording as much information as possible continuously, during the test, is critical. Coupling that with the ability to reduce the landing energies to low and ultra-low landing energies enables comparative surface studies, to the nanometer scale [1] as well as correlated imaging [2].

In this study, in situ comparative heating and simultaneous multi-detector imaging at high and ultra-low landing energies were examined to various type of conductive and non-conductive materials.

Figure 1 shows the SEM instrument and MEMS heating holder used for the study. The Hitachi SU7000 variable pressure field emission scanning electron microscope with a Schottky thermionic emission source was used for the study. The SU7000 has capability to simultaneously display and detect multiple signals at one working distance. The Blaze heating holder, utilizing a custom MEMS heating chip (Norcada) [3] designed for Hitachi SU7000 stage was used for the heating experiment and control. The maximum heating temperature is $\sim 1100{ }^{\circ} \mathrm{C}$ and is fully digitally controlled by the SEM operator.

Figure 2a shows simultaneous multi-detector imaging results of Au particles on a Si substrate at 3 different temperature points at 40,375 and $550^{\circ} \mathrm{C}$, which are extracted from the entire in situ comparative heating test shown in the chart data (Figure 2b). UD detector collects secondary electrons showing fine surface morphology on the Si substrate and Au particles. PDBSE Compo shows backscattered electron and crystal information of the Au including Si impurities that disappear at $550^{\circ} \mathrm{C}$. PDBSE Custom setting highlights the topographic information. PDBSE custom image enhances the porosity developed at $375^{\circ} \mathrm{C}$ then grown at $550{ }^{\circ} \mathrm{C}$ in two of the particles.
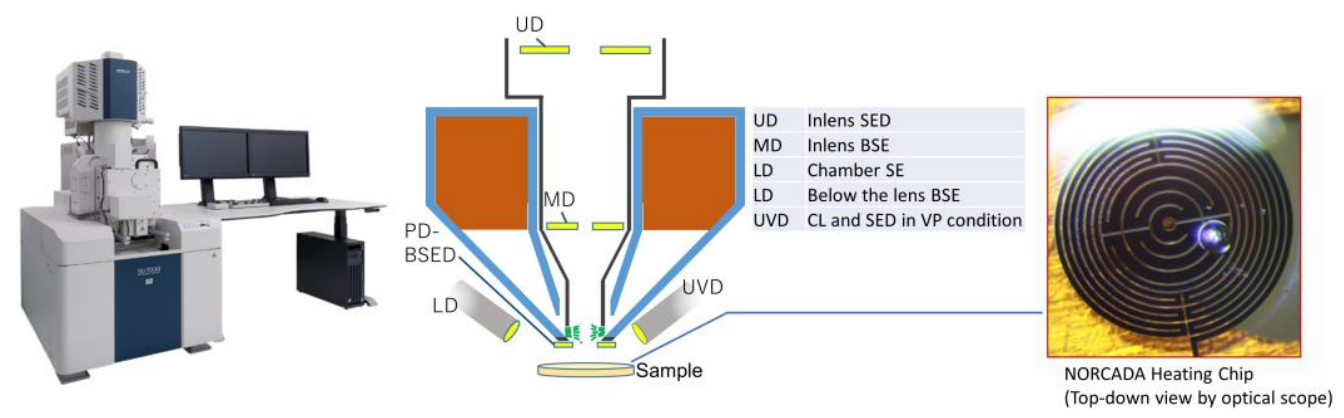

Figure 1. Figure 1. Hitachi SU7000 FE-SEM, schematics of detector configuration and MEMS heating chip. 

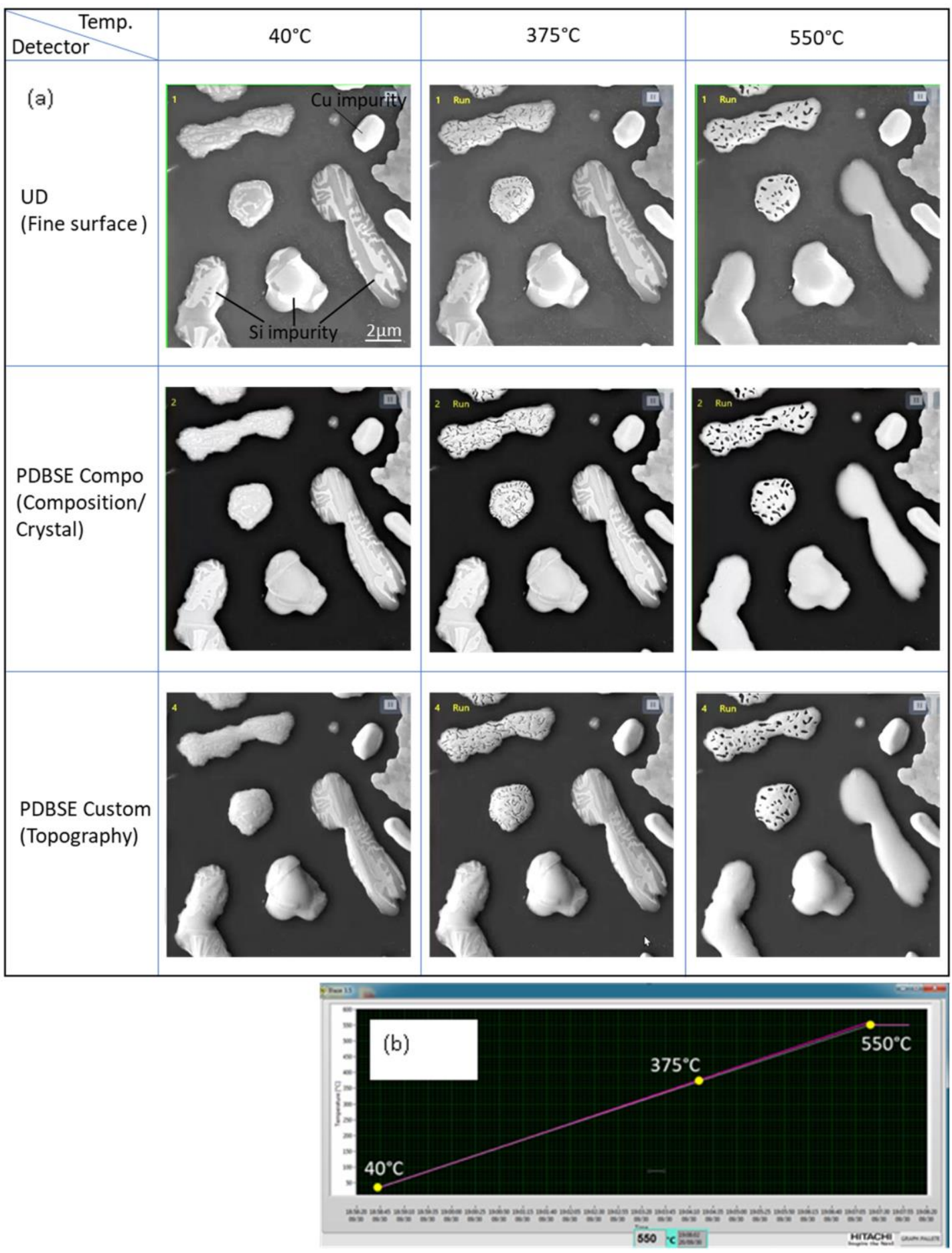

Figure 2. Figure 2. In situ heating and simultaneous imaging of Au particles on Si: (a) Multi detector images extracted at 40,375 and $550^{\circ} \mathrm{C}$ (b) Heating chart window with showing data extraction points

References

[1] M Postek et al, Proc. SPIE. 11467(2020) 114670Q

[2] Y Hashimoto et al, Microsc. Microanal. 25 (Suppl 2) (2019) p.p.564

[3] J Howe et al, Microsc. Microanal. 23 (Suppl 1) (2017) p.p.66 